\title{
APROXIMACIÓN A LA CARACTERIZACIÓN DEL EMPRENDIMIENTO FEMENINO: UNA INVESTIGACIÓN CUALITATIVA EN CLAVE COMPETENCIAL
}

\author{
APPROACH TO THE CHARACTERIZATION OF FEMALE ENTREPRE- \\ NEURSHIP: A QUALITATIVE RESEARCH IN COMPETENCE KEY
}

\author{
Ángel José Olaz Capitán \\ Pilar Ortiz García \\ Universidad de Murcia, Murcia. España/Spain \\ olazcapi@um.es \\ portizg@um.es
}

Recibido/Received: 21/04/2017

Modificado/Modified: 24/04/2017

Aceptado/Accepted: 29/09/2017

\section{RESUMEN}

El propósito de este trabajo es identificar qué elementos inhiben el espíritu empresarial entre las mujeres. En este análisis se ha utilizado la técnica de grupo nominal como herramienta de investigación cualitativa con el fin de establecer estrategias y acciones que reviertan esta situación. A través de sesiones de trabajo, las participantes han establecido un conjunto de estrategias que minimicen estas dificultades y se han seleccionado los proyectos clave para el desarrollo de la iniciativa empresarial innovadora en las mujeres a partir de competencias.

\section{PALABRAS CLAVE}

Competencias; Mujer; Género, Emprendimiento; Técnica de Grupo Nominal.

\section{SUMARIO}

1. Introducción. 2. La conexión entre mujer, emprendimiento y competencias. 3. Aspectos metodológicos. 4. Análisis de resultados. 5. Propuestas de actuación. 6. Cuestiones competenciales. 7. Conclusiones. Bibliografía.

\begin{abstract}
The purpose of this paper is to identify which elements inhibit entrepreneurship among women. In this analysis we have used the Nominal Group Technique as a tool for qualitative research in order to establish strategies and actions to revert this situation. Through working sessions, participants have established a set of strategies that would allow minimizing these difficulties and they have selected key projects for developing innovative entrepreneurship in women through competencies.
\end{abstract}

\section{KEYWORDS}

Competencies; Woman; Gender; Entrepreneurship; Nominal Group Technique. 


\section{CONTENTS}

1. Introduction. 2. The connection between women, entrepreneurship and competences. 3 . Methodological aspects. 4. Analysis of results. 5. Proposals for action. 6. Competencial issues. 7. Conclusions. References.

\section{INTRODUCCIÓN}

Este trabajo tiene por propósito reflexionar sobre la caracterización del emprendimiento femenino en términos competenciales. La oportunidad, relevancia e interés de este tema queda justificado por la posición de la mujer respecto al trabajo, una posición que adquiere con gran frecuencia la consideración de actividad complementaria, lo que explicaría una mayor irregularidad en las trayectorias laborales de las mujeres en un "mundo de hombres".

Este trabajo forma parte de la investigación "Mujer y Emprendimiento desde una Perspectiva Competencial” (CSO2013 - 43667 - R) - financiado por el Ministerio de Economía y Competitividad- en el que en su vertiente cualitativa se ha recurrido a la Técnica de Grupo Nominal. A través de esta técnica y mediante muestras estructurales se han identificado los elementos inhibidores del emprendimiento, por razón de género, y se ha planteado por parte de las participantes estrategias de partida y actuaciones orientadas a revertir esta situación concretándolas en competencias profesionales con valor de mercado,

Las siguientes líneas introducirán, en primer lugar, algunas cuestiones relativas y elementos contextualizadores que permiten vertebrar el trinomio mujer - emprendimiento competencias, a continuación se hablará sobre la lógica del proceso metodológico relacionado con esta herramienta y más tarde se comentará la explotación de los datos, análisis de resultados y algunas conclusiones preliminares de este proyecto de investigación.

\section{LA CONEXIÓN ENTRE MUJER, EMPRENDIMIENTO Y COMPETENCIAS}

Existe un carácter diferencial en el emprendimiento por razón de género como lo demuestran estudios relevantes en esta materia (Ruiz et al, 2012), si bien es cierto que según los datos del GEM para 2012, para España, se dan ciertas concordancias entre el perfil de hombres y mujeres emprendedores, por ejemplo en lo relativo a edad, en torno a los 38 años y en cuanto al núcleo familiar de procedencia, compuesto por un número ligeramente superior a los tres miembros, no obstante, se aprecian diferencias en otros aspectos tales como el nivel de estudios y de renta, así como en el origen geográfico. En cuanto al primero de los aspectos, la formación, el Informe GEM señala que las mujeres suelen tener una formación ligeramente superior a la de los emprendedores varones. En concreto, en 2012 el porcentaje de emprendedoras con un nivel de estudios universitario es de $38,4 \%$ frente al $32,3 \%$ de este mismo nivel en el caso de los hombres.

También hay que constatar la diferencia entre ambos sexos en cuanto al nivel de renta, en este caso, a favor de los emprendedores varones, un hecho que el Informe GEM atribuye al tipo de empresa que crean unos y otras y que, en el caso de las mujeres, tiene un carácter más convencional, menos tecnológico y más orientada al consumo.

El emprendimiento femenino contribuye, por una parte, a impulsar el crecimiento económico a partir de la creación de empresas y, por otra, a reforzar las políticas de igualdad. 
La importancia adquirida por esta actividad se pone de manifiesto en las investigaciones sobre el tema, centradas en analizar los factores que condicionan el emprendimiento por razón de género y, especialmente, las razones que explican la menor participación de la mujer en este proceso (Kelley et al., 2011).

Por otro lado, la perspectiva competencial supone una contribución inicial en el avance de la investigación sobre el tema sobre el que no existe una literatura tan abundante como en los aspectos anteriormente reseñados. Bajo estos elementales supuestos, esta investigación provoca tres grandes líneas de análisis. Son las siguientes:

\subsection{La actividad empresarial en la mujer}

La caracterización de la actividad empresarial de las mujeres, así como el tipo de empresas creadas (innovación, potencial de crecimiento, tamaño y sector) es una premisa necesaria en el análisis competencial de la actividad emprendedora. Respecto a la actividad empresarial femenina, se manifiesta como una importante fuente de crecimiento económico (Allen et al., 2007; De Bruin, et al., 2006 Macaulay, 2003).

Otros autores ponen de manifiesto las menores oportunidades de las mujeres hacia puestos que impliquen responsabilidad y toma de decisiones. (Gisbert et al., 2009; Mateos et al., 2009; Peris - Ortiz et al., 2010; Burke, 2007)

En cuanto a la tipología de empresas creada por mujeres, la literatura establece una relación entre las características del negocio (tamaño, sector, innovación, internacionalización y financiación) y el éxito o fracaso de la actividad emprendedora (Estrin y Mickiewicz, 2011; Ruiz et al., 2012), así como su repercusión sobre la visibilidad de la actividad empresarial femenina (Anna et al., 2000; Du Rietz y Henrekson, 2000; Bird y Sapp, 2004; Orser et al., 2006).

\subsection{El emprendimiento femenino}

Gran parte de la literatura sobre el tema en cuestión ha puesto de manifiesto que, a pesar de las iniciativas institucionales orientadas a la puesta en marcha de la actividad emprendedora femenina ésta, sin embargo, se mantiene en unas tasas de participación inferiores a las de los hombres (Kelley et al. (2011). La cautela y prevención de la mujer posiblemente como resultado de ciertas construcciones sociales - ayuda a explicar, el menos en parte, esta realidad. Concretamente algunos autores mencionan factores del entorno relacionados con el carácter sociocultural del espacio en el que las personas se desenvuelven (Verheul et al., 2001; Busenitz et al., 2003; Steyaert y Katz 2006; Alvarez y Urbano, 2011), aspectos institucionales, como el acceso a la financiación y otras redes sociales de colaboración (Alsos et al., 2006; Carter et al., 2007; Gatewood et al., 2009; Kim, 2006; Marlow y Patton, 2005).

A estas cuestiones deben añadirse otras percepciones que enlazan con ciertos factores informales - percepción de las competencias y habilidades que las personas tienen o creen tener - relevantes para el emprendimiento femenino. En este sentido, resultan reveladoras algunas investigaciones sobre el tema (Bruni et al., 2004; Álvarez et al., 2012) que establecen una relación entre aspectos de esta naturaleza y los componentes diferenciales del emprendimiento en función del género.

\subsection{Los aspectos competenciales}

Las competencias - y de un modo especial, para la mujer emprendedora - son la traducción formal de la cualificación exigible y autoexigida para el desarrollo del proyecto emprendedor. 
Pero ¿cómo puede definirse una competencia? McClelland (1973) define inicialmente el término competencia como algo que realmente causa un rendimiento superior en el trabajo, anteponiendo su significado y dimensión al enfoque educativo, para ponerlo en correspondencia con otros elementos alternativos como el género, etnia o clase social para medir el rendimiento laboral de la persona en el contexto organizativo.

Casi de foma coincidente en el tiempo Bloom (1975) menciona que la "enseñanza basada en competencias", se asienta sobre la idea de que todo aprendizaje es esencialmente individual; el individuo, al igual que cualquier sistema, se orienta hacia la consecución de un conjunto de metas a lograr y el proceso de aprendizaje es más fácil cuando el individuo sabe qué es exactamente lo que se espera de él.

Según Boyatzis (1982) las competencias pueden consistir en: a) Motivos, b) Rasgos de carácter o predisposición general a conducirse o reaccionar de modo determinado; c) Concepto de uno mismo o lo que uno piensa, lo que valora y/o lo que está interesado a realizar; d) Conocimientos o lo que se sabe sobre una técnica, ciencia y/o habilidad y e) Capacidades cognitivas y de conducta, ya sean ocultas (razonamiento deductivo) u observables (escucha activa).

En suma, un conjunto de matizaciones orientadas a una mejor definición del término competencia que tiempo más tarde no pasan inadvertidas a Lawler (1994) a quien se le debe su aplicabilidad práctica al mundo empresarial o la idea de cómo las ventajas de pasar a una empresa gestionada por competencias, implica el paso de la burocratización a un modelo sistémico - organizativo.

En nuestro país, los trabajos realizados por Pereda y Berrocal (2001) inspirados en las ideas de Le Boterf et al. (1993) describen cinco elementos que, en su opinión, ayudan a definir la naturaleza de la competencia. Estos elementos se corresponderían con cinco tipos de saberes: a) Saber o conocimientos que posee la persona; b) Saber Hacer o la capacidad que tiene esa persona para aplicar aquellos conocimientos; c) Saber Estar o la realización de esos comportamientos en función de los procedimientos de la organización; d) Querer hacer $\mathrm{y}$, finalmente; e) Poder hacer o las características organizativas que permiten al individuo disponer de los medios y recursos para desarrollar su competencia.

Ya más recientes en el tiempo, son los estudios de naturaleza epistemo - metodológica de Haro (2004), que en un intento por clasificar la infinidad de modelos realiza una propuesta basada en la comprensión del término competencia, según sea entendida como variable dependiente o independiente.

Es en este segundo caso, al ser caracterizada como variable independiente, la competencia es contemplada como causa u origen del desempeño o resultado. A su vez, las causas que determinan el desempeño eficaz y eficiente de los trabajadores son de varios tipos: el desempeño que se basa en rasgos, en conductas o en una combinación de ambos. De este modo, concluye señalando cómo la competencia es un constructo con el que se califican comportamientos relacionados entre sí, siendo éstos los responsables directos de un resultado excelente en el desempeño del puesto de trabajo.

Olaz (2011) enfatiza en 3 grandes dimensiones de plena coincidencia con el espíritu emprendedor: conocimientos, capacidades y habilidades. La primera dimensión guarda relación con los conocimientos (reglados o no reglados) con los que una persona cuenta desde una perspectiva teórica - práctica. Estos conocimientos pueden estar legitimados, bien desde la formación reglada, o desde los aspectos informales que se recogen en las vivencias y experiencias que jalonan la vida cotidiana. Existe una segunda dimensión vinculada a las capacidades, entendidas como el potencial relacionado con las características naturales del individuo para gestionar determinadas situaciones. Es importante precisar que, en principio, 
si bien todo el mundo nace con estas, ello no comporta un desarrollo uniforme en el mismo rango de individuos, lo que apunta a la influencia de aspectos ecológicos y ambientales para explicar las diferencias existentes en su desarrollo. Ejemplos de esta dimensión pueden ser: a) la motivación por el logro, la preocupación por el orden y la claridad, la iniciativa y proactividad, y el autocontrol personal, por citar sólo algunos. La tercera dimensión, en algunos casos colindante con la anterior, se refiere a ciertas destrezas no necesariamente innatas, ni tan siquiera sospechadas por el propio individuo, que al ser "descubiertas" por necesidad del puesto de trabajo permiten un mejor desarrollo a la persona en su relación con el entorno. Ejemplo de ellas son la dirección de reuniones, resolución de conflictos y capacidad negociadora y planificación de actividades, entre otras.

En esta línea de trabajo, resulta determinante cotejar las competencias auto - asignadas por parte de las mujeres y las competencias que demanda el mercado, no siempre coincidentes. Son varios los estudios que contemplan la competencia como causa u origen del desempeño o resultado de la actividad laboral (De Haro, 2004; Brändle y Olaz, 2013). Las investigaciones de Langowitz y Morgan (2003) y de Langowitz y Minnitti (2007), muestran que las mujeres emprendedoras se perciben como menos validas con relación a los hombres. Estos estudios sugieren que mientras que los hombres tienden a emprender por factores relacionados con la oportunidad, la financiación o el deseo de eliminar una frustración profesional, las mujeres lo hacen debido a una situación laboral precaria o por las dificultades de acceso a un empleo, en otras palabras, por necesidad propia.

De este modo, se potencia entre los hombres la auto - percepción de competencias para emprender, mientras que entre las mujeres se fomenta una auto-percepción negativa hacia dicha actividad (Brush, 1992; Marlow, 2006; Dolinsky y Caputo, 2003).

\section{ASPECTOS METODOLÓGICOS}

La Técnica de Grupo Nominal - traducción del término anglosajón Nominal Group Technique (N.G.T.) - fue dada a conocer en 1.968 y se atribuye su creación principal a Delbecq y Van de Ven (1975), con objeto de mejorar el desarrollo de reuniones de trabajo y su dinamización operativa buscando la productividad exigible a las mismas.

Habría que esperar a 1981 a que Rohrbaugh aludiera explícitamente bajo el término de Técnica de Grupo Nominal.

Esta es una herramienta orientada al diagnóstico, interpretación e intervención en la realidad social, sin embargo, dentro del conjunto de técnicas de investigación cualitativas no es de las más conocidas, posiblemente debida a cierta complejidad metodológica - sólo posiblemente - en relación a otras más asentadas como las entrevistas cualitativas (Vallés, 2007), los grupos de discusión (Ibáñez, 1986), (Ortí, 1989) y la observación participante (Hernández Pedreño y Sabater, 2015) por citar sólo algunas.

Entre los principales objetivos diferenciadores de está técnica de investigación cualitativa caben señalar los siguientes:

1. Constituir un ambiente propicio y una atmósfera adecuada para que el grupo de trabajo se convierta en un "laboratorio de ideas" destinado a proporcionar nuevas dimensiones de análisis para el estudio, desde una óptica creativa.

2. Obtener diferentes puntos de vista relacionados con el escenario estudiado, desde la perspectiva del grupo en un intento por conocer el estado de la cuestión.

3. Generar un proceso que basado en datos e informaciones permita extraer conclusiones sobre las posibles causas, elementos condicionantes y determinantes del fenómeno estudiado. 
4. Analizar las vinculaciones existentes entre los diferentes aspectos considerados, identificando las posibles relaciones causales existentes.

5. Configurar un grupo de trabajo que permita llegar al nivel de consenso suficiente para, a partir de ese instante, obtener el compromiso con los asistentes.

6. Trazar estrategias y tácticas operativas que permitan modelizar la puesta en marcha de soluciones concretas y adaptadas a la problemática estudiada.

7. En niveles mas avanzados por el contenido, alcance y dimensión del proyecto constituir un "observatorio" del fenómeno estudiado en el transcurso del tiempo.

8. Complementar a otras técnicas de investigación cuantitativas en el estudio de los fenómenos desde la visión que proporciona un pluralismo metodológico.

\subsection{Muestra y Perfiles seleccionados}

Como en numerosos estudios de naturaleza cualitativa y por impedimentos relacionados con el conocimiento del universo poblacional se ha recurrido a muestras estructurales que, si bien no comparten los requisitos de una muestra estadística, permiten explorar las representaciones y construcciones sociales que adornan a los colectivos en su particular interpretación de la realidad social.

En este trabajo nos centraremos en 2 sesiones realizadas, la primera en Murcia Ciudad (21/05/2015) y la otra en Lorca (01/06/2015), distante de la anterior a $60 \mathrm{Kms}$. El perfil de las mujeres empresarias era similar en ambas localidades, perteneciendo en su gran mayoría al sector terciario, con una edad entre los 35 y 50 años, con menos de 10 empleados y con una experiencia emprendedora de 3.5 años. Bajo esta caracterización inicial se desarrolló el protocolo durante las cerca de 3.5 horas que duró cada sesión.

El interés por examinar el diagnóstico, interpretación y apuesta por acciones concretas por parte de cada uno de los 2 colectivos puede verse influido por las características socioeconómicas del grupo a (Murcia ciudad) frente a las del grupo b (Lorca). Mientras que en el primero el carácter urbanita, profesionalizado y quizás más motivado por el sentido de la oportunidad a la hora de acometer el emprendimiento, se alejaba del radicado en Lorca, a caballo entre el carácter urbanita y el rural, curiosamente menos terciarizado, algo menos profesionalizado (debido a una menor cualificación formativa) y marcado por un emprendimiento en que la necesidad primaba sobre el sentido de la oportunidad a la hora de acometer el emprendimiento.

\subsection{Breve descripción del proceso}

El proceso genéricamente se desarrolla a partir de la formulación de la siguiente pregunta ¿Qué aspectos dificultan, deterioran, impiden o limitan el emprendimiento femenino? Esta cuestión principal suscita otros tantos interrogantes relacionados que indirectamente han de ser contestados.

El proceso comienza con una breve reflexión individual se organizan una serie de grupos de trabajo (equipos autónomos de trabajo) que discuten y priorizan sus contestaciones en unas cartulinas de diferentes colores según el peso explicativo de la respuesta $\left(1^{\mathrm{er}}\right.$ orden de importancia, $2^{\circ}$ orden, etc.) Sobre esta base, el investigador, con el acuerdo de los equipos, agrupa las contestaciones en diferentes escenarios.

Tras este primer diagnóstico la dinámica discurre por una fase en la que se pondera el peso explicativo de cada aspecto, atendiendo al número de tarjetas (ponderación bruta) y al valor asociado a cada tarjeta según el color asignado (ponderación relativa).

En esta fase el equipo de trabajo en su conjunto, procederá a establecer todos los cruces posibles entre los diferentes escenarios, sin olvidar que cada uno de ellos es origen y causa a 
la vez o, si se prefiere, variable dependiente y / o independiente de las otras consideradas y hasta del elemento central de estudio.

Como resultado de esta secuencia, el equipo de trabajo aborda de qué manera y bajo que acciones concretas qué medidas se van a tomar, qué instrumentos y herramientas se van adoptar, quién, dónde y cuándo se encargará de materializar estas medidas para revertir aquellos aspectos que han deteriorado, impedido o limitado el emprendimiento femenino.

\section{ANÁLISIS DE RESULTADOS}

Tras este recorrido por el proceso anterior y en un intento por homogeneizar escenarios entre las 2 muestras estructurales de Murcia ciudad y Lorca, los escenarios representados son los que pueden observarse en la Tabla 1.

Tabla 1: Escenarios

\begin{tabular}{|c|c|c|c|c|}
\hline Murcia & $\begin{array}{c}\text { Aspectos } \\
\text { Psicológicos }\end{array}$ & $\begin{array}{c}\text { Aspectos } \\
\text { Sociales / } \\
\text { Familiares }\end{array}$ & $\begin{array}{c}\text { Aspectos } \\
\text { Educativos }\end{array}$ & $\begin{array}{c}\text { Aspectos } \\
\text { Culturales }\end{array}$ \\
\hline $\begin{array}{c}\text { Ponderación } \\
\text { relativa }\end{array}$ & $43.0 \%$ & $19.0 \%$ & $10.0 \%$ & $27.0 \%$ \\
\hline
\end{tabular}

\begin{tabular}{|c|c|c|c|c|}
\hline Lorca & $\begin{array}{c}\text { Aspectos } \\
\text { Psicológicos }\end{array}$ & $\begin{array}{c}\text { Aspectos } \\
\text { Sociales / } \\
\text { Familiares }\end{array}$ & $\begin{array}{c}\text { Aspectos } \\
\text { Educativos }\end{array}$ & $\begin{array}{c}\text { Aspectos } \\
\text { Económicos / } \\
\text { Financieros }\end{array}$ \\
\hline $\begin{array}{c}\text { Ponderación } \\
\text { relativa }\end{array}$ & $18.4 \%$ & $40.2 \%$ & $18.4 \%$ & $23.0 \%$ \\
\hline
\end{tabular}

Fuente: Elaboración propia

La ponderación relativa representa en términos porcentuales el peso que los participantes han concedido a cada uno de estos aspectos en contestación a la pregunta: ¿Qué aspectos dificultan, deterioran, impiden o limitan el emprendimiento femenino?

Es interesante advertir que los aspectos psicológicos, los sociales y familiares y, también, los educativos son comunes a los dos grupo, mientras que los aspectos culturales (en Murcia ciudad) y los económico - financieros (en Lorca) son propios de cada espacio geográfico.

En Murcia ciudad los aspectos psicológicos - o personales - del individuo (43.0\%) son los que mayor peso explicativo tienen a la hora de explicar los posibles inhibiciones en el emprendimiento femenino, mientras que los familiares y sociales son menores $(19.0 \%)$, justo casi al contrario que en el grupo de Lorca - población con tintes más rurales que urbanos -, donde los aspectos inhibidores del emprendimiento pasan por contemplar los escenarios familiares (40.2\%), mientras que los psicológicos solo llegan al 18.4\%. Estos datos refuerzan la idea de cómo lo "cosmopolita" va más asociado a la idea del individualismo, mientras que los lazos familiares condicionan y hasta determinan las decisiones de las personas en ámbitos menos urbanitas y más rurales. En el caso de Lorca, caso representativo de una localidad en que el sector primario es clave en su desarrollo económico - social local, los aspectos educativos (formativos) son más valorados en cuanto a cómo su ausencia puede tener más repercusiones en el emprendimiento que en 
una gran ciudad como es Murcia, en la que la formación se supone a priori más elevada ( $18.4 \%$ en la primera y $10.0 \%$ en la segunda).

Por último, resaltar los aspectos disonantes en cada localidad. Mientras que en Murcia ciudad la ausencia de referentes culturales (contactos, redes, capital relacional) retrae el emprendimiento, en el caso de Lorca, estos aspectos - quizás por su propio tamaño - están más asentados. En la T.G.N. realizada en Lorca, los elementos inhibidores del emprendimiento tienen su referencia en los aspectos económico - financieros, sin los cuales resultaría complejo la puesta en marcha de un modelo de negocio.

\subsection{Comentarios relacionados con la Sesión realizada en Murcia}

Entre los principales aspectos limitadores del emprendimiento destacan los aspectos psicológicos con un peso explicativo del $43.0 \%$. Aspectos como: el exceso de responsabilidad, la dependencia y carga emocional - más veces proviniente de los hijos que del cónyuge - puede ir asociada en situaciones límite al sentimiento de culpa, unido a un exacerbado sentido de la responsabilidad y autoexigencia personal - profesional, configuran parte del mosaico que retrata el perfil muestral de la mujer emprendedora. Otros aspectos como el miedo al rechazo, el fracaso y la huída ente el conflicto también contribuyen a explicar las auto - limitaciones que se impone el colectivo femenino en un contexto de trazas machistas, donde la competitividad con el hombre es elevada y la edad interviene como elemento modulador de su actuación. Queda por analizar si estos aspectos calificados de "psicológicos" por las intervinientes pueden ser el resultado de una construcción social osmotizada e interiorizada desde la socialización primaria

Los aspectos sociales y familiares con una ponderación del $19.0 \%$ indican como la educación familiar y los tópicos que en esta se instalan, configuran una base sobre la que la mujer debe actuar en el siempre dificultoso y penoso intento por intentar conciliar - en general ella sola, exista o no cónyuge - la vida personal con la profesional. Cuando la crinza de los hijos es tarea exclusiva de la mujer y existe ausencia de apoyos el tema se complejiza más si cabe, lo que redunda en dificultades para gestionar el tiempo. Por último, otro elemento inhibidor del emprendimiento de la mujer es el realacionado con las dificultades para acceder a fuentes de financiación y como, en determinados ambientes, la solicitud de crédito puede verse mermada no tanto por las características del emprendimiento como por la percepción de género y la percepción (machista) que se tiene sobre la capacidad de la mujer para llevar a cabo determinados procesos.

Por último, los aspectos educativos con un $10.0 \%$ de poder explicativo aluden a cuestiones de algún modo reseñadas transversalmente en anteriormente. Educación y formación (reglada o no reglada) son piezas claves en el proceso de modelización del emprendimiento, pero no lo es menos la importancia por "combatir" el machismo de la mujer como principal foco de actuación para evitar y revertir las sucesivas construcciones de género que llevan a situaciones discriminadoras en una teórica sociedad desarrollada.

Los aspectos culturales con un peso explicativo del $27.0 \%$, señalan como elementos inhibidores del emprendimiento femenino, el omnipresente liderazgo masculino - lógico y propio de un mundo de hombres y para hombres - y los escrúpulos relacionados con ciertos valores y códigos éticos que abarcan diferentes planos (también el sexual) como expresión de diferentes sensibilidades, valores y comportamientos más responsables. De nuevo, los roles de género, fomentados desde edades tempranas en el ámbito familiar condicionan y determinan desde la elección de los estudios al desarrollo profesional que más tarde se haya decidido proyectar $\mathrm{y}$, en clara correspondencia, hasta el tipo de emprendimiento. 


\subsection{Comentarios relacionados con la Sesión realizada en Lorca}

El área "envolvente" de esta ciudad es eminentemente agrícola - ganadera, pero la localidad y quizás más, después del terremoto (11/05/2011), parece haberse reorientado hacia al sector servicios.

Entre los principales aspectos limitadores del emprendimiento se encuentran los aspectos psicológicos con un $18.4 \%$. Estos aspectos psicológicos vinculados a lo emocional, la incertidumbre - expresada como tal en su conjunto - junto al miedo al fracaso en todas sus componentes (personales, familiares, sociales...) son cuestiones que concitan la atención de las mujeres emprendedoras, sin olvidar las dificultades en lo referente a la delegación de funciones que muy posiblemente se encuentra ligada a una mal percibida autosuficiencia.

Los aspectos sociales y familiares (40.2 \%) no escapan al diagnóstico de las mujeres emprendedoras donde la invisibilidad es un elemento central de análisis. En otras palabras, el desconocimiento que de ellas se tiene, de su propia capacidad y de su propio negocio es un primer freno a la hora de darse a conocer. Existe, por tanto, una necesidad de establecer redes que visibilicen y pongan en valor el proyecto empresarial más allá del propio entorno en el que actúan. Un entorno inmediato en el que la imagen física puede verse alterada por la condición de ser persona extranjera. Este sentimiento larvado que presupone la inferioridad de la mujer y pone bajo sospecha su capacidad empresarial, puede conducir a situaciones, donde el miedo al fracaso y / o al ridículo termina por ralentizar y hasta "invitar" al cese de la actividad y, lo que es peor, impedir el desarrollo de un proyecto que además de económico tiene elevados componentes vitales.

Otros aspectos igualmente "denunciados" son los derivados de la maternidad, la crianza de los hijos - también el cuidado y atención a familiares como padres y mayores - que demandan un tiempo precioso, dificultan la conciliación con la vida personal y la empresarial. Estas cuestiones unidas a ciertas percepciones de género y al rol familiar que la mujer ha de desempeñar hacen que la sombra de la familia sea alargada y perdurable en el tiempo. Quedaría por analizar con más detalle, si la proximidad a un área rural, contribuye a amplificar una visión tradicional de las cuestiones de género en las que la dicotomía entre funciones productivas y reproductivas parecen seguir latentes.

En tercer lugar los aspectos educativos con un $18.4 \%$ de peso explicativo es un tema clave cuando existen necesidades de formación. Las asistentes señalan como elementos inhibidores el desconocimiento del mundo empresarial al que se ven abocadas - esto apuntaría hacia el emprendimiento por necesidad - la falta de experiencia laboral (que sólo se forja en el día a día), junto a una deficiente formación académica (reglada) en temas relacionados con la organización de los recursos y en gestión general.

En cuarto lugar emerge un nuevo escenario que no aparecía en la sesión realizada en Murcia. Son los aspectos económicos - financieros $(23.0 \%)$ y como el acceso a la concesión del crédito sigue siendo un elemento clave en la comprensión de la génesis del emprendimiento. Las elevadas cargas que la mujer debe asumir - hasta en las formas más sencillas del emprendimiento - como el "darse de alta como autónoma", siguen siendo algo oneroso, lo que unido a la situación de crisis, y las siempre exigentes condiciones que suponen reembolsar el capital e intereses, dificultan el inicio y sostenimiento de la actividad. Frente a esta opción quedaría como alternativa el apoyo familiar que, en ocasiones, no posee los recursos suficientes para avalar este tipo de proyectos por pequeña que sea la inversión a realizar. 


\section{PROPUESTAS DE ACTUACIÓN}

Tras este análisis una de las aportaciones fundamentales de esta técnica es la posibilidad de apelar al grupo - ahora transformado en pequeños equipos autónomos de trabajo (EE.AA.TT.) para el diseño y puesta en marcha de acciones que minoren, modulen, atemperen $\mathrm{o}$, incluso, hasta reviertan, los elementos inhibidores del emprendimiento femenino. La tabla 2 recoge una síntesis de las acciones propuestas por los intervinientes en las 2 sesiones celebradas.

Tabla 2: Acciones Propuestas

\begin{tabular}{|l|l|}
\hline Aspectos & Soluciones / Propuestas \\
\hline (1) & $\begin{array}{l}\text { Gestionar el tiempo y las emociones. } \\
\text { Delegar tareas y responsabilidades. } \\
\text { Adquirir técnicas para el autoconvencimiento y motivación con los éxitos } \\
\text { registrados. } \\
\text { Aprender de los fracasos y transformarlos en oportunidades } \\
\text { Desarrollar técnicas de relajación y autocontrol. } \\
\text { "Educar" a la familia implicando a los miembros en el desarrollo profesional } \\
\text { de la mujer. } \\
\text { Organizar el tiempo y planificación de Recursos. }\end{array}$ \\
\hline Fijarse objetivos a corto plazo. \\
Formarse para paliar el miedo al fracaso e incertidumbre y ganar en \\
Foutosuficiencia.
\end{tabular}

Fuente: Elaboración propia 
Del resultado de este tipo de análisis, los diferentes EE.AA.TT. proponen y desarrollan este conjunto de mejoras en el intento de llevarlas a la práctica en dimensiones espacio temporales concretas. Evidentemente no todas pueden materializarse de un modo inmediato con los recursos propios por lo que se impone una priorización de las mismas y, todavía más importante que eso, adquirir el compromiso de impulsarlas.

\section{CUESTIONES COMPETENCIALES}

Despues de lo visto en páginas precedentes, hablar de competencias es hablar de un conjunto de elmentos cualificadores de distinta naturaleza que dotan al individuo, en nuestro caso, emprendedor - en su concepto génerico - de "resortes" que vehiculizan su potencial.

De la importante literatura sobre emprendimiento no existen una igual abundancia de estudios sobre su carácter competencial. Según revela el estudio de Raičević et al. (2007) entre las competencias para el empresario emprendedor destacan las actitudes estratégicas para el emprendimiento y la propia actividad empresarial. Entre las primeras, actitudes estratégicas, se incluyen: disposición a mostrar iniciativa, actitud positiva a cambiar e innovar y voluntad para identificar áreas donde se puede evidenciar las habilidades empresariales.

Si resulta complejo recurrir a estudios contrastados sobre el carácter emprendedor desde una perspectiva competencial, no lo es menos el contar con estudios donde el género femenino es su elemento central de análisis, salvo interesantes excepciones donde se realiza una comparativa entre hombres y mujeres.

Ruiz et al. (2012: 53 - 55), en su análisis sobre la Actividad Emprendedora de las mujeres en España (2005 - 2011), señala tres grandes bloques competenciales (que dan lugar a otras tantas competencias significativas). Son los siguientes: Autoconfianza emprendedora, Tolerancia al riesgo y el reconocimiento de oportunidades. Del resultado de este trabajo cabe observar que se aprecia cierta diferencia significativa, aunque no especialmente acusada, entre hombres y mujeres, a favor de los primeros, en cuanto a autoconsiderarse capacitados para emprender (autoconfianza emprendedora). En esta dirección, los porcentajes de mujeres que manifiestan que el temor al fracaso es un obstáculo vital para la creación de empresas, son siempre superiores a los registrados en el caso de los hombres. Estos elementos condicionan la propensión a emprender, lo que unido a la percepción subjetiva relacionada con el reconocimiento de oportunidades de negocio, en la población masculina, constata que la mujer es mucho más precavida además de los aspectos competenciales por razones de índole educativa, familiar, social y hasta ideológica.

El estudio de Ventura y Quero (2013) sobre factores explicativos de la intención de emprender en la mujer, revela otras competencias tales como a) Valoración del resultado; b) Autoconfianza; c) Antecedentes familiares; d) Capital social y e) Intención de emprender como aspectos competenciales significativos del emprendimiento femenino.

Más recientemente el estudio de ASEME (2015) sobre las causas de la escasa representación de las mujeres empresarias en determinados sectores y segmentos del tejido empresarial de la Comunidad de Madrid, revela igualmente un conjunto muy interesante de compentencias como son: a) Deseo de Independencia; b) Asunción de riesgos; c) Carácter positivo y emprendedor; d) Motivaciones personales relacionadas con los conocimientos del sector o conocimiento del mismo; e) Creatividad y Carácter Innovador; f) Habilidades en gestión y administración (autoeficacia); g) Relaciones Interpersonales reforzadas, Habilidades Sociales y Proactividad; h) Integración en empresa de familiares / amigos / 
conocidos.

Por último y conforme al proceso descrito llegó el momento en el que tras diagnosticar los elementos inhibidores del emprendimiento femenino y desarrollar un conjunto de acciones que pudieran revertir estos condicionantes, se imponía el traducir estas acciones a competencias.

El modelo empleado se basa en el denominado ECI (Emotional Competence Inventory) de Hay Group McClelland Center for Research and Innovation (2005)

El ECI ha mostrado la validez del constructo competencial en diferentes ámbitos como por ejemplo en lo referente a la intuición y dimensiones de pensamiento - sentimiento (introversión y extraversión) (Burckle, 2000). Este modelo ha sido también empleado a la hora de correlacionarlo con el Clima Laboral (Sala, 2003), Inteligencia emocional (Stubbs, 2005) y Creencias irracionales de los individuos (Welpe,Tumasjan, Stich et al., 2005). También deben mencionarse dos estudios examinaron la validez discriminante de la ECI con respecto a otros instrumentos de medición (Murensky, 2000) con relación al Pensamiento Crítico y también el de Byrne (2003), concluyendo la idoneidad en la arquitectura del constructo.

Este modelo se basa en 4 dimensiones que engloban a 18 competencias. Las dimensiones son las siguientes:

Dimensión 1: recoge las competencias relacionadas con el autoconocimiento personal. Proporciona información sobre el grado de conocimiento emocional del individuo, así como de las fortalezas y debilidades de carácter y cómo ello repercute en el nivel de autoconfianza.

Dimensión 2: se orienta a la detección de las pulsiones del individuo en términos de fijación de objetivos y metas - logros -, capacidad para tomar la iniciativa ante las oportunidades; coherencia; adaptabilidad a los cambios y actitud positiva ante los acontecimientos vitales.

Dimensión 3: identificar en el individuo los principios de alteridad. Se trata de una de las competencias clave, especialmente cuando de trabajo se trata, ya que posibilita la interpretación de los estados de ánimo, prioridades y expectativas del grupo, lo que resulta de suma utilidad en el trabajo en equipo.

Dimensión 4: indaga en los aspectos que tienen que ver con la gestión de las relaciones interpersonales, desde la capacidad de liderazgo de iniciativas y cambio organizacional, hasta la evaluación de las competencias para trabajar en equipo, pasando por las habilidades persuasivas de la persona. Todas ellas configuran una de las dimensiones clave para el desarrollo del individuo en las organizaciones.

Tras un proceso de diálogo con los asistentes a las sesiones de T.G.N. se les solicitó que determinarán cuáles eran a su juicio las competencias que de un mejor modo podrían actuar como elementos aceleradores de las mejoras conducentes a revertir aquellas cuestiones que limitaban o inhibían el proceso emprendedor de la imagen. Del resultado de esta parte del proceso, la tabla 3 , sintetiza los resultados de las dos sesiones realizadas.

\section{CONCLUSIONES}

Como en muchos estudios de naturaleza cualitativa la configuración de una muestra estructural viene determinada por la identificación y selección de contextos relevantes al problema de investigación. Esto comporta observar ese difícil equilibrio entre la accesibilidad y el grado de heterogeneidad - homogeneidad de los individuos que componen la muestra y que están condicionados a la explotación de redes personales y, en su caso, a los 
"contactadores" disponibles.

Desde esta perspectiva este trabajo de investigación ha pretendido comprender el enfoque de los actores desde la perspectiva de su ecosistema, analizar el modo en que éstos perciben, interiorizan y viven sus realidades concretas. En definitiva, el objetivo no es tanto rescatar las vivencias íntimas de los sujetos en proceso, como comprender los significados que guían sus acciones puesto que tales acciones contribuyen a la construcción de la realidad social.

El emprendimiento, algo en principio ajeno a la cuestión de género, sin embargo, introduce un conjunto de reflexiones motivadas por el papel que las mujeres pueden desempeñar en un mundo de hombres y para hombres.

Esto comporta que los factores inhibidores para el emprendimiento, en el caso de la mujer, estén teñidos de dificultades adicionales al caso de los hombres que, de darse, no son de tal calado. Las razones de base tienen que ver con el hecho de que el emprendimiento parece contextualizarse como una ocupación complementaria que debe simultanearse con el solícito papel de hija, esposa y madre.

Tabla 3: Competencias potenciadoras de la inhibición del emprendimiento

\begin{tabular}{|c|c|c|c|c|}
\hline Aspectos & $\begin{array}{l}\text { Dimensión } 1 \\
\text { Autoconocimiento } \\
\text { personal }\end{array}$ & $\begin{array}{l}\text { Dimensión } 2 \\
\text { Autogestión }\end{array}$ & $\begin{array}{l}\text { Dimensión } 3 \\
\text { Conciencia social }\end{array}$ & $\begin{array}{l}\text { Dimensión } 4 \\
\text { Gestión de las relaciones }\end{array}$ \\
\hline $\begin{array}{l}\text { (1) } \\
\text { Psicológicos }\end{array}$ & $\begin{array}{l}\text { 1. Conciencia } \\
\text { emocional } \\
\text { 2. Autoevaluación } \\
\text { 3. Autoconfianza }\end{array}$ & \begin{tabular}{|l|} 
4. Autocontrol \\
Emocional \\
5. Transparencia \\
7. Logro \\
8. Iniciativa \\
9. Optimismo \\
\end{tabular} & 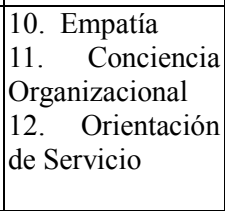 & $\begin{array}{l}\text { 13. Desarrollo de personas } \\
\text { 14. Liderazgo inspirador } \\
\text { 16. Influencia } \\
\text { 17. Gestión de conflictos } \\
18 \text { Trabajo en equipo }\end{array}$ \\
\hline $\begin{array}{l}(2) \\
\text { Sociales } \\
\text { familiares }\end{array}$ & 3. Autoconfianza & \begin{tabular}{|l|} 
4. Autocontrol \\
Emocional \\
5. Transparencia \\
6. Adapatbilidad \\
7. Logro \\
9. Optimismo \\
\end{tabular} & $\begin{array}{l}\text { 10. } \\
\text { 12. Ompatía } \\
\text { de Serviciontación }\end{array}$ & $\begin{array}{l}\text { 13. Desarrollo de personas } \\
\text { 15. Catalizar el cambio } \\
\text { 16. Influencia } \\
\text { 17. Gestión de conflictos } \\
\text { 18. Trabajo en equipo }\end{array}$ \\
\hline $\begin{array}{l}\text { (3) } \\
\text { Educativos }\end{array}$ & $\begin{array}{l}\text { 2. Autoevaluación } \\
\text { 3. Autoconfianza }\end{array}$ & $\begin{array}{l}\text { 5. Transparencia } \\
\text { 7. Logro }\end{array}$ & $\begin{array}{l}\text { 10. } \\
\text { 12. Ompatía } \\
\text { de } \text { Serviciontación }\end{array}$ & $\begin{array}{l}\text { 13. Desarrollo de personas } \\
\text { 14. Liderazgo inspirador } \\
\text { 15. Catalizar el cambio } \\
\text { 16. Influencia } \\
\text { 17. Gestión de conflictos } \\
\text { 18. Trabajo en equipo }\end{array}$ \\
\hline $\begin{array}{l}4) \\
\text { Culturales } \\
\end{array}$ & - & - & - & $\begin{array}{l}\text { 15. Catalizar el cambio } \\
\text { 16. Influencia }\end{array}$ \\
\hline $\begin{array}{l}5) \\
\text { Aspectos ec. } \\
\text { y financieros }\end{array}$ & - & 6. Adaptabilidad & - & 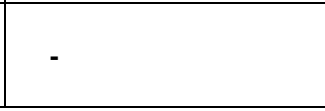 \\
\hline
\end{tabular}

Fuente: Elaboración propia

Estas cuestiones unidas al emprendimiento por necesidad (complementar los ingresos de la economía doméstica) más que por oportunidad, algo más propio de los hombres, y una menor cualificación formativa que en el género masculino motiva que este emprendimiento sea más vulnerable y de menor recorrido.

Los aspectos psicológicos como potenciales inhibidores del emprendimiento de la mujer ayudarían a entender como la falta de confianza es clave en la comprensión del 
emprendimiento. No debe olvidarse que en los aspectos personales también influyen los del entorno y cómo estos pueden ser determinantes en la confianza y motivación de la mujer.

Otros aspectos igualmente reseñables son aquellos relacionados los aspectos educativos (formativos) que en el caso de mujeres, en el tramo de los 45 a 65 años, quedan por debajo de los hombres, algo propio de sociedades donde el papel de la mujer estaba destinado a los quehaceres domésticos.

Es pues el momento de actuar desde un enfoque competencial como suma de algo más que los tradicionales conocimientos, sino además de las capacidades y habilidades de las personas, sin olvidar que el autoconocimiento de la persona y la autogestión son puntos de partida irrenunciable.

\section{BIBLIOGRAFÍA}

Alsos, G.; Isaksen, E. y Ljunggren, E. (2006) "New Venture Financing and Subsequent Business Growth in Men- and Women - Led Businesses". Entrepreneurship Theory y Practice, 5: 667 - 686.

Álvarez, C.; Noguera, M y Urbano, D. (2012) "Condicionantes del entorno y emprendimiento femenino. Un estudio cuantitativo en España. Economía Industrial, 383: 43-51.

Alvarez, C. y Urbano, D. (2011) "Una decada de investigación sobre el GEM: logros y retos". Academia Revista Latinoamericana de Administración, 46: 16 - 37.

Allen, I.E.; Elam, A.; Langowitz, N.; Llen, I.E.; Elam, A.; Langowitz, N. y Dean, M. (2007) Report on Women and Entrepreneurship. Global Entrepreneurship Monitor. Massachusetts, USA: The Center for Women's Leadership at Babson College,

Anna, A.L.; Chyler G.N.; Jansen, E. y Mero, N.P. (2000) "Women business owners in traditional and non - traditional industries". Journal of Business Venturing, 15 (3): 279 -303.

ASEME (2015) Causas de la escasa representación de las Mujeres empresarias en determinados sectores y segmentos del tejido empresarial de la Comunidad de Madrid., en http://www.aseme.es/empresas/aseme/Causas_infra.pdf [consulta 21/04/2017].

Bird, S. y Sapp, S. (2004) "Understanding the gender gap in small business success: Urban and Rural comparisons". Gender and Society, 18 (1): 5 - 28.

Bloom, S.(1975) Evaluación del aprendizaje. Buenos Aires: Troquel.

Boyatzis, R. (1982) The competence manager. New York: John Wiley \& Sons.

Busenitz, L.W.; West, G.P.; Shepherd, D.; Nelson, T.; Chandler, G.N. y Acharakis, A. (2003) "Entrepreneurship research in emergence: Past trends and future directions". Journal of Management, 29 (3): 285 - 308.

Brändle, G. y Olaz, A. (2013) "Diseño de una entrevista de evaluación del desempeño por competencias desde una perspectiva microsociológica". Aposta, (58): 1- 32

Bruni, E.A.; Gherardi, S. y Poggio, P. (2004) "Entrepreneur - mentality, gender and the study of women entrepreneurs". Journal of Organizational Change Management, 17 (3): 256 - 268.

Burckle, M. (2000) ECI and MBTI, Hay/McBer Research Report. Boston: Hay / McBer Group.

Brush, C.G. (1992) "Research on women business owners: Past trends, a new perspective and future directions". Entrepreneurship Theory and Practice, 16 (4): 5 - 31.

Byrne, J. C. (2003) The role of emotional intelligence in predicting leadership and related work behavior. Hoboken: Stevens Institute of Technology, Technology Management.

Carter, S.; Shaw, E.; Lam, W. y Wilson F. (2007) "Gender, Entrepreneurship, and Bank Lending: The Criteria and Processes Used by Bank Loan Officers in Assessing Applications". Entrepreneurship Theory y Practice, 31 (3): 427 - 444.

De Bruin, A.; Welter, F. y Brush, C.G. (2006) "Introduction to special issue: Advancing Cumulative Knowledge on Women's Entrepreneurship". Entrepreneurship Theory y Practice, 30(5): 585 - 593.

Delbecq, A. y Van De Ven, A. (1975) Group techniques for program planning: A guide to nominal group and Delphi processes. Glenview, IL.: Scott - Foresman \& Co.

Dolinsky, A.L. y Caputo, R.K. (2003) "Health and female selfemployment". Journal of Small Business 
Management, 41(3): 233 - 241.

Du Rietz, A. y Henrekson, M. (2000) "Testing the Female Underperformance Hypothesis". Small Business Economics, 14 (1): 1 - 10.

Estrin, S. y Mickiewicz, T. (2011) "Institutions and female entrepreneurship". Small Business Economics, 37 (4): 397 - 415.

Gatewood, E.J.; Brush, C.; Carter, N.; Greene, P. y Hart, M. (2009) "Diana: a symbol of women entrepreneurs' hunt for knowledge, money, and the rewards of entrepreneurship". Small Business Economics, 32 (2): 129 - 145.

GEM (2012). Informe Global Entrepreneurship Monitor (2012) España 2012, Madrid, Ministerio de Industria, Energía y Turismo.

Gisbert, M.C.; Alarcón, M.J. y Gómez, J.M. (2009) "La mujer y su papel en la industria. Aproximación al caso del sector del juguete". Economía Industrial, 372: 51- 64.

Haro (De), J. (2004) “¿Sabe alguien qué es una competencia?”. Dirigir personas, 30: 8 - 17.

Hay Group Mcclelland Center For Research and Innovation (2005) Emotional Competence Inventory (ECI) Technical Manual. Prepared by Steven B. Wolff, DBA. Updated November 2005.

Hernández Pedreño, M. y Sabater, C. (2015) "La observación participante como técnica de investigación social”. En N. Caparrós y E. Raya, E. (Coords.) Métodos y Técnicas de Investigación en Trabajo Social. Madrid: Grupo 5, pp. 175 - 192.

Ibáñez, J. (1986) "Cómo se realiza una investigación mediante grupos de discusión". En M. García Ferrando; J. Ibáñez, y F. Alvira (Coords.). El Análisis de la Realidad Social. Métodos y Técnicas de Investigación Social. Madrid: Alianza Universidad Textos, pp. 418-434

Kelley, D.J. ; Bosma, N. y Amorós, J.E. (2011) Global Entrepreneurship Monitor 2010 Global Report. Londres: Global Entrepreneurship Research Association, accesible en: https://goo.gl/TUEjQh

Kim, G.O. (2006) "Do Equally Owned Small Businesses Have Equal Access to Credit?" Small Business Economics, 27 (4-5): $369-386$.

Langowitz, N.S. y Morgan, C. (2003) "Women entrepreneurs: breaking through the glass barrier" En J. Butler (Ed.) New perspectives on women entrepreneurs. Greenwich CT: Information Age Publishing, pp. $101-119$.

Langowitz, N.S. y Minniti, M. (2007) "The Entrepreneurial Propensity of Women". Entrepreneurship Theory and Practice, 31 (3): 341 - 364.

Lawler, E. (1994) "From Job - Based to Competence - Based Organizations". Journal of Organizational Behavior, 15 (1): 3 - 15.

Le Boterf, G.; Vincent, F. y Barzucchetti, S. (1993) Cómo gestionar la calidad de la formación. Barcelona: Gestión 2000.

Macaulay, C. (2003) "Changes to Self-Employment in the UK: 2002 to 2003". Labour Market Trends, December: $623-628$.

McClelland, D. (1973) "Testing for competence rather than for intelligence". American Psychologist, 28(1): $13-20$.

Marlow, S. (2006) "A safety net or ties that bind? Women, welfare and self - employment". International Journal of Sociology y Social Policy, 26 (9 - 10): 397 - 410.

Marlow, S. y Patton D. (2005). “All Credit to Men?" Entrepreneurship, Finance, and Gender, Entrepreneurship Theory y Practice, 29 (6): 717 - 735.

Mateos, R.; Iturrioz, J. y Gimeno, R. (2009) "La participación financiera y el papel de la mujer en la toma de decisiones de las sociedades cooperativas: los consejos de administración". Revista europea de dirección y economía de la empresa 18(3): 65 - 82.

Olaz, A. (2011) "Una aproximación conceptual a la cualificación profesional desde una perspectiva competencial". Papers, 96 (2): $589-616$

Orser, B.J.; Riding, A.L. y Manley, K. (2006) "Women Entrepreneurs and Financial Capital". Entrepreneurship Theory \& Practice, 30 (5): 643 - 645.

Ortí, A. (1989) "La apertura y el enfoque cualitativo o estructural: la entrevista abierta, semidirigida y la discusión de grupo". En M. García Ferrando, J. Ibáñez y F. Alvira (Coords.) El Análisis de la Realidad Social. Métodos y Técnicas de Investigación Social. Madrid: Alianza Universidad Textos, pp. $171-204$. 
Pereda, S. y Berrocal, F. (2001) Gestión de recursos humanos por competencias. Madrid: Centro de estudios Ramón Areces.

Peris - Ortiz, M.; Peris, F. y Ribeiro, D. (2010) "Capacidades y mujer emprendedora". Información Comercial Española, 852: 153 - 165.

Raičević, S.; Šćekić, D.; Vučurović,V. y Jaćimović, Ž. (2007) Key competences for lifelong learning. Development of key competences in the Montenegrin education system. Torino: European Training Foundation.

Rohrbaugh, J. (1981) "Improving the quality of group judgement: Social judgment analysis and the Nominal Group Technique". Organizational Behavior and Human Performance, 28: 272 - 288.

Ruiz, J.; Coduras, A. y Camelo, M. (2012) Actividad Emprendedora de las mujeres en España. Madrid: Ministerio de Sanidad, Servicios Sociales e Igualdad.

Sala, F. (2003) "Leadership in education: effective UK college Principals". Nonprofit Management \& Leadership, 14(2): 171-189.

Steyaert, C. y Katz, J. (2004) "Reclaiming the space of entrepreneurship in society: Geographical, discursive and social dimensions". Entrepreneurship y Regional Development, 16 (3): 179 - 196.

Stubbs, C. E. (2005) Emotional intelligence competencies in the team and team leader: A multi-level examination of the impact of emotional intelligence on group performance. Unpublished Dissertation. Cleveland: Case Western Reserve University, Organizational Behavior.

Vallés, M. (2007) Entrevistas Cualitativas, Madrid, Centro de Investigaciones Sociológicas.

Ventura, R. y Quero, M. (2013) "Factores explicativos de la intención de emprender en la mujer. Aspectos diferenciales en la población universitaria según la variable género". Cuadernos de Gestión, 13 (1): 135-155.

Verheul, I. y Thurik, R. (2001) "Start-up capital: does gender matter?" Small Business Economics, 16 (4): 329 - 345.

Welpe, I.; Tumasjan, A.; Stich, J.; Spörrle, M. y Försterling, F. (2005) "Emotional Intelligence and its consequences for occupational and life satisfaction - Emotional Intelligence in the context of irrational beliefs". 47th conference of experimentally working psychologists. Lengerich: Pabst Science Publishers.

\section{Breve currículo:}

\section{Ángel José Olaz Capitán}

Doctor en Sociología por la Universidad de Murcia y licenciado en Ciencias Económicas por la Universidad Autónoma de Madrid. En la actualidad desarrolla su actividad docente e investigadora en la Universidad de Murcia donde imparte la asignatura métodos y técnicas de investigación social. Es investigador principal del proyecto Mujer y Emprendimiento desde una Perspectiva Competencial (CSO2013 - 43667 - R) y del proyecto Discapacidad y Emprendimiento. Análisis Competencial (CSO2016-75818-R), financiados por el Ministerio de Economía y Competitividad (Gobierno de España).

\section{Pilar Ortiz García}

Doctora en Ciencias Económicas por la Universidad de Murcia y licenciada en Sociología por la Universidad Complutense de Madrid. Profesora titular del Departamento de Sociología de la Facultad de Economía y Empresa de la Universidad de Murcia donde imparte la asignatura sociología del trabajo. Es investigador principal del proyecto Mujer y Emprendimiento desde una Perspectiva Competencial (CSO2013 - 43667 - R) y del proyecto Discapacidad y Emprendimiento. Análisis Competencial (CSO2016-75818-R), financiados por el Ministerio de Economía y Competitividad (Gobierno de España). 